\title{
Density functional theory
}

\author{
Maylis Orio - Dimitrios A. Pantazis • \\ Frank Neese
}

Received: 15 November 2008/Accepted: 12 January 2009/Published online: 24 February 2009

(C) The Author(s) 2009. This article is published with open access at Springerlink.com

\begin{abstract}
Density functional theory (DFT) finds increasing use in applications related to biological systems. Advancements in methodology and implementations have reached a point where predicted properties of reasonable to high quality can be obtained. Thus, DFT studies can complement experimental investigations, or even venture with some confidence into experimentally unexplored territory. In the present contribution, we provide an overview of the properties that can be calculated with DFT, such as geometries, energies, reaction mechanisms, and spectroscopic properties. A wide range of spectroscopic parameters is nowadays accessible with DFT, including quantities related to infrared and optical spectra, X-ray absorption and Mössbauer, as well as all of the magnetic properties connected with electron paramagnetic resonance spectroscopy except relaxation times. We highlight each of these fields of application with selected examples from the recent literature and comment on the capabilities and limitations of current methods.
\end{abstract}

Keywords Density functional theory . Spectroscopic properties - Photosystem .

Oxygen evolving complex

M. Orio · D. A. Pantazis · F. Neese $(\bowtie)$

Lehrstuhl für Theoretische Chemie, Institut für Physikalische und Theoretische Chemie, Universität Bonn, Wegelerstrasse 12,

53115 Bonn, Germany

e-mail: neese@thch.uni-bonn.de

M. Orio · D. A. Pantazis - F. Neese

Max-Planck-Institut für Bioanorganische Chemie,

Stiftstrasse 34-36, 45470 Mülheim an der Ruhr, Germany

\section{Introduction}

The present contribution is devoted to the use of density functional theory (DFT) in bioinorganic chemistry and more specifically in the modeling of structures, properties, and processes related to photosynthesis. DFT has been established as a valuable research tool because it can serve either to validate the conclusions that have been reached from the analysis of the experiments or to distinguish between those possibilities that were left open. The calculation of a wide range of molecular properties with DFT allows a close connection between theory and experiment and often leads to important clues about the geometric, electronic, and spectroscopic properties of the systems being studied. Here, we will first introduce briefly the general theoretical principles that constitute the basis of the DFT approach. Our priority in this paper is to describe what can be achieved through the practical use of DFT; therefore, we will then focus on the properties that can be computed and mention selected applications of DFT for molecular property calculations, drawn principally from examples relevant to photosynthetic research. We will comment not only on the strengths but also on the technical pitfalls and the current limitations of the technique, discussing the performance of DFT and the foreseeable achievements in the near future.

\section{Theoretical background}

To appreciate the special place of DFT in the modern arsenal of quantum chemical methods, it is useful first to have a look into the more traditional wavefunction-based approaches. These attempt to provide approximate solutions to the Schrödinger equation, the fundamental equation of 
quantum mechanics that describes any given chemical system. The most fundamental of these approaches originates from the pioneering work of Hartree and Fock in the 1920s (Szabo and Ostlund 1989). The HF method assumes that the exact $N$-body wavefunction of the system can be approximated by a single Slater determinant of $N$ spinorbitals. By invoking the variational principle, one can derive a set of $N$-coupled equations for the $N$ spin orbitals. Solution of these equations yields the Hartree-Fock wavefunction and energy of the system, which are upper-bound approximations of the exact ones. The main shortcoming of the HF method is that it treats electrons as if they were moving independently of each other; in other words, it neglects electron correlation. For this reason, the efficiency and simplicity of the HF method are offset by poor performance for systems of relevance to bioinorganic chemistry. Thus, HF is now principally used merely as a starting point for more elaborate "post-HF" ab initio quantum chemical approaches, such as coupled cluster or configuration interaction methods, which provide different ways of recovering the correlation missing from HF and approximating the exact wavefunction. Unfortunately, postHF methods usually present difficulties in their application to bioinorganic and biological systems, and their cost is currently still prohibitive for molecules containing more than about 20 atoms.

Density functional theory attempts to address both the inaccuracy of HF and the high computational demands of post-HF methods by replacing the many-body electronic wavefunction with the electronic density as the basic quantity (Koch and Holthausen 2000; Parr and Yang 1989). Whereas the wavefunction of an $N$ electron system is dependent on $3 N$ variables (three spatial variables for each of the $N$ electrons), the density is a function of only three variables and is a simpler quantity to deal with both conceptually and practically, while electron correlation is included in an indirect way from the outset. Modern DFT rests on two theorems by Hohenberg and Kohn (1964). The first theorem states that the ground-state electron density uniquely determines the electronic wavefunction and hence all ground-state properties of an electronic system. The second theorem establishes that the energy of an electron distribution can be described as a functional of the electron density, and this functional is a minimum for the groundstate density. Thus, the problem of solving the many-body Schrödinger equation is bypassed, and now the objective becomes to minimize a density functional. Note, however, that although the Hohenberg-Kohn theorems assure us that the density functional is a universal quantity; they do not specify its form.

In practice, the common current realization of DFT is through the Kohn-Sham (KS) approach (Kohn and Sham 1965a). The KS method is operationally a variant of the HF approach, on the basis of the construction of a noninteracting system yielding the same density as the original problem. Noninteracting systems are relatively easy to solve because the wavefunction can be exactly represented as a Slater determinant of orbitals, in this setting often referred to as a Kohn-Sham determinant. The form of the kinetic energy functional of such a system is known exactly and the only unknown term is the exchange-correlation functional. Here lies the major problem of DFT: the exact functionals for exchange and correlation are not known except for the free electron gas. However, many approximations exist which permit the calculation of molecular properties at various levels of accuracy.

The most fundamental and simplest approximation is the local-density approximation (LDA), in which the energy depends only on the density at the point where the functional is evaluated (Kohn and Sham 1965b). LDA, which in essence assumes that the density corresponds to that of an homogeneous electron gas, proved to be an improvement over HF. While LDA remains a major workhorse in solid state physics, its success in chemistry is at best moderate due to its strong tendency for overbinding. The first real breakthrough came with the creation of functionals belonging to the so-called generalized gradient approximation (GGA) that incorporates a dependence not only on the electron density but also on its gradient, thus being able to better describe the inhomogeneous nature of molecular densities. GGA functionals such as BP86 (Becke 1988) or PBE (Perdew et al. 1996) can be implemented efficiently and yield good results, particularly for structural parameters, but are often less accurate for other properties. The next major step in the development of DFT was the introduction of hybrid functionals, which mix GGA with exact Hartree-Fock exchange (Becke 1993). Nowadays, hybrid DFT with the use of the B3LYP functional (Becke 1988; Lee et al. 1988) is the dominant choice for the treatment of transition metal containing molecules (Siegbahn 2003). This method has shown good performance for a truly wide variety of chemical systems and properties, although specific limitations and failures have also been identified.

More recent theoretical and methodological developments include the "meta-GGA" functionals, which extend the GGA corrections to higher derivatives, and the "double hybrid" functionals (Grimme 2006a, b; Neese et al. 2007a), which contain not only a fraction of exact exchange but also a fraction of orbital-dependent nonlocal correlation energy estimated at the level of second-order many-body perturbation theory. These new functionals, such as TPSSh (Staroverov et al. 2003) and B2PLYP (Grimme 2006a, b), respectively, yield improved energetics and spectroscopic properties, and will likely see more use in the future as their performance and range of applicability is established. 


\section{Properties and applications}

Geometries

Optimizing the geometry of the species under investigation is the first step in most theoretical studies. Geometries predicted by DFT tend to be quite reliable and the optimized structures usually agree closely with X-ray diffraction (XRD) or extended X-ray absorption fine structure (EXAFS) data. From our experience, the achievable accuracy for short and strong metal-ligand bonds is excellent, whereas intra-ligand bonds are predicted typically within 2 pm of experiment. Weaker metal-ligand bonds are usually overestimated by up to 5 pm (Neese 2006a, b). A reasonable choice of basis set has to be made, although this condition does not pose particularly stringent requirements since the structures predicted by all DFT methods generally converge quickly with basis set size, thus making geometry optimization rather economical. Basis sets of valence triple$\zeta$ quality plus polarization are usually enough to get almost converged results for geometries; however, results obtained with smaller basis sets should be viewed with caution. An extended study of the performance of various modern functionals and basis sets for the geometries of all first-, second-, and third-row transition metals has recently appeared (Bühl et al. 2008). Weak interactions are not satisfactorily treated with current density functionals owing to the wrong asymptotic behavior of the exchange-correlation potential, but this deficiency can be overcome to some extent by inclusion of functional-specific empirical dispersion corrections (Grimme 2006a, b).

Concerning the choice of method, the differences between density functionals are usually small for structural parameters making the choice of functional not critical for the success of a geometry optimization. GGA functionals provide good geometries and are sometimes even better than hybrid functionals, which also tend to be more expensive (Neese 2006a, 2008a). The computational efficiency of GGA in practical applications stems from the density fitting approximation (Baerends et al. 1973; Vahtras et al. 1993; Eichkorn et al. 1997) that is implemented in many quantum chemistry programs and significantly speeds up GGA calculations. This allows for fast optimizations, an important advantage especially when many different probable structures have to be considered. This has been the case, for example, in a recent computational study focusing on possible models of the oxygen evolving complex (OEC) in photosystem II (PSII), which used $\mathrm{Mn}_{4} \mathrm{O}_{x}$ topologies derived from accurate polarized EXAFS measurements (Yano et al. 2005). The great number of possible protein ligation patterns and the additional potential for a multitude of protonation and hydration states (Fig. 1) creates the need for efficient geometry optimizations which can be

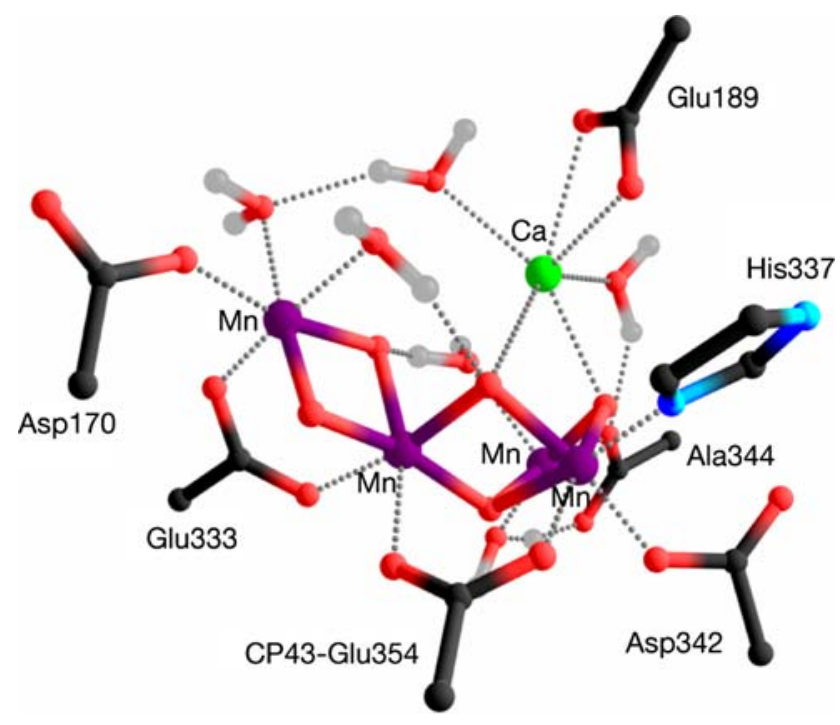

Fig. 1 Optimized geometry of an OEC model constructed on top of a polarized EXAFS topology for the $\mathrm{Mn}_{4} \mathrm{O}_{5} \mathrm{Ca}$ cluster; side-chain and water ligation shown are one out of many possibilities (Zein et al. 2008a)

performed with GGA functionals such as BP86. Once optimized structures have been obtained, other molecular properties can be evaluated using a potentially more accurate hybrid functional (Zein et al. 2008a). Exploring many structural alternatives and their corresponding spectroscopic properties in this way is an important step in crossvalidating theory and experiment, forming the basis for further elaboration toward more realistic models.

Despite the overall good performance of GGA functionals, it is still likely that for certain systems high accuracy can be achieved only with hybrid functionals. In this case, the obvious choice has traditionally been the B3LYP functional. More recent studies, however, have accumulated evidence that the hybrid PBE0 and TPSSh functionals are superior performers for systems within the field of inorganic and bioinorganic chemistry (Bühl et al. 2008; Jensen 2008), the latter yielding improved energies as well. The particularly promising performance of TPSSh has been attributed in part to the use of $10 \%$ exact exchange, a value half-way between GGA and B3LYP (20\%). It should be noted at this point that the computational disadvantage of hybrid functionals mentioned earlier will likely be diminished with the arrival of new state-of-the-art and potentially linear-scaling procedures such as the 'chain of spheres' (COSX) approximation to HF exchange (Neese et al. 2008).

Energetics and reaction mechanisms

Locating transition state structures is a more complicated task for the researcher, but in many ways it is computationally the same as optimizing a geometry; the difference is simply that the target now is not a minimum 
on the potential energy surface but rather a saddle point. Once this stationary point is found and its energy is computed, one gains immediate access to energy barriers and is therefore able to study reaction mechanisms. However, if this effort is to have any real value, the calculated relative energies must be reasonably accurate. A great number of studies over the years have converged to the conclusion that energetic predictions with the B3LYP functional tend to be systematically more accurate and reliable than GGA functionals. Hence, this hybrid functional is widely used for predicting and/or elucidating the major features of various mechanisms in bioinorganic chemistry (Siegbahn 2006b). The estimated error of B3LYP is approximately 2-3 kcal mol${ }^{-1}$ for the G2 reference set of molecules, but this figure is probably too optimistic when one is faced with electronically complicated open-shell transition metal systems like those encountered in almost any bioinorganic setting. It is safe to say that there is no consensus regarding the optimal choice of method when one considers additionally the prediction of energies for electronically distinct states of the same species, such as those arising from different electronic configurations of a metal center, from a different distribution of oxidation states within a metal cluster, or even from the interplay between metal-centered and ligand-centered redox processes. When these factors come into play, the error margin can easily exceed by far the optimistic range mentioned earlier.

Nevertheless, even if the estimated errors may be already too large for quantitative predictions in cases of small activation energies such as those observed during the catalytic cycle of the OEC (Sproviero et al. 2007), the simulation of reaction pathways is a fundamentally important application of DFT. A representative example that stands out in the field of photosynthesis research is the systematic work that has been focused on elucidating mechanistic aspects in the catalytic cycle of OEC (Lundberg and Siegbahn 2004; Siegbahn 2006a, 2008a, b; Sproviero et al. 2008a, b). This line of work demonstrates that DFT calculations can offer significant input to mechanistic investigations, sometimes revealing possibilities that were not previously considered. It should be kept in mind, however, that a reaction mechanism predicted by DFT cannot be validated on the basis of computed energies alone, especially when the structure of the principal component is itself debatable. All such efforts should attempt to combine and incorporate many lines of evidence, taking into account additional criteria such as the spectroscopic properties of the putative intermediates.

Vibrational frequencies

Closely connected in research practice to the procedure of structural optimization is the calculation of vibrational frequencies. They are used not only for simulating infrared (IR) or Raman spectra but also for characterizing the nature of stationary points as minima or transition states. Moreover, the information obtained from such a calculation is used to compute statistical thermodynamic corrections to the electronic energy and thus to make direct comparisons with experimentally determined free energies. It is well established that the predicted harmonic frequencies with GGA functionals such as BP86 and PBE typically agree well with measured vibrational fundamentals if basis sets of polarized triple- $\zeta$ quality are used (Murray et al. 1992; Sosa et al. 1992; Stratmann et al. 1997). It has been shown, however, that this excellent accuracy is a result of error cancelation between the neglect of anharmonicity and systematic errors in the prediction of the correct harmonic frequencies (Neugebauer and Hess 2003). We note that the effects of anharmonicity are practically impossible to be computed with DFT for large systems of interest to biology. Intensities of IR as well as Raman modes can, however, be obtained straightforwardly.

Theoretical studies on a model of the oxygen evolving complex of PS II have demonstrated how computed vibrational frequencies can provide valuable feedback for the interpretation of experimental data. Specifically, calculations by Gascon et al. (2007) suggested that the vibrational modes of carboxylate groups ligated to manganese ions of the OEC might be insensitive to changes in the formal oxidation states of the ions because of electron delocalization within the cluster. At the same time, it was shown that the charge rearrangement associated with the Sstate transitions in the OEC might induce shifts in the vibrational frequency of carboxylate groups that do not function as direct ligands to the manganese ions. These theoretical results imply that the vibrational frequency shifts observed in experimental FTIR measurements do not necessarily have to be interpreted as reflecting changes in the first coordination sphere of the Mn cluster, thus providing ways to reconcile the perceived discrepancies between FTIR and XRD data (Sproviero et al. 2008b).

\section{Optical spectra}

Density functional theory is restricted from its foundations to ground states only; therefore, the calculation of excited states and their properties has to be approached indirectly. This is achieved using time-dependent linear response theory, in which one studies the frequency dependence of a time-dependent electric field perturbation, the poles of which provide excitation energies. Thus, time-dependent DFT (TD-DFT) calculations yield the transition energy rather than the total energy of the excited state, which therefore is never explicitly calculated (Bauernschmitt and Ahlrichs 1996; Casida et al. 1998; Stratmann et al. 1998). 
It should be noted that the TD-DFT approach allows also for a full determination of the central quantities involved in the calculation of both absorption and circular dichroism (CD) spectra. It is also possible to predict magnetic circular dichroism (MCD) spectra through TD-DFT calculations (Seth et al. 2004, 2005; Seth and Ziegler 2006), although $a b$ initio multireference approaches are preferred in this respect since they explicitly cover the correct physics involved (Ganyushin and Neese 2008).

Optical spectra predicted by TD-DFT with the use of either the BP86 or B3LYP functionals may occasionally be of acceptable quality (Fiedler et al. 2005; Jackson et al. 2005; Schenker et al. 2005; Stich et al. 2005) even though many problematic cases and a multitude of artifacts plague this methodology (Grapperhaus et al. 2001; Neese 2008a). TD-DFT problems arise principally from the shortcomings of current functionals and include the erroneous treatment of states with ionic or charge transfer character, poor prediction of highly excited Rydberg states, entirely missing states when double excitations are involved, and the inability to obtain the correct multiplet structures for openshell systems. In general, one has to apply TD-DFT calculations with utmost caution and it is imperative to seek critical feedback from experimental data. With this provision, TD-DFT can be a useful interpretative tool, as was recently demonstrated by Sun et al. (2007) in their study of the P700 system found in the reaction center (Fig. 2) of photosystem I (PSI). The authors used TD-DFT in conjunction with the statistical average of different orbital potentials (SAOP) model (Gritsenko et al. 1999) to examine the excitation processes in the pair of chlorophylls

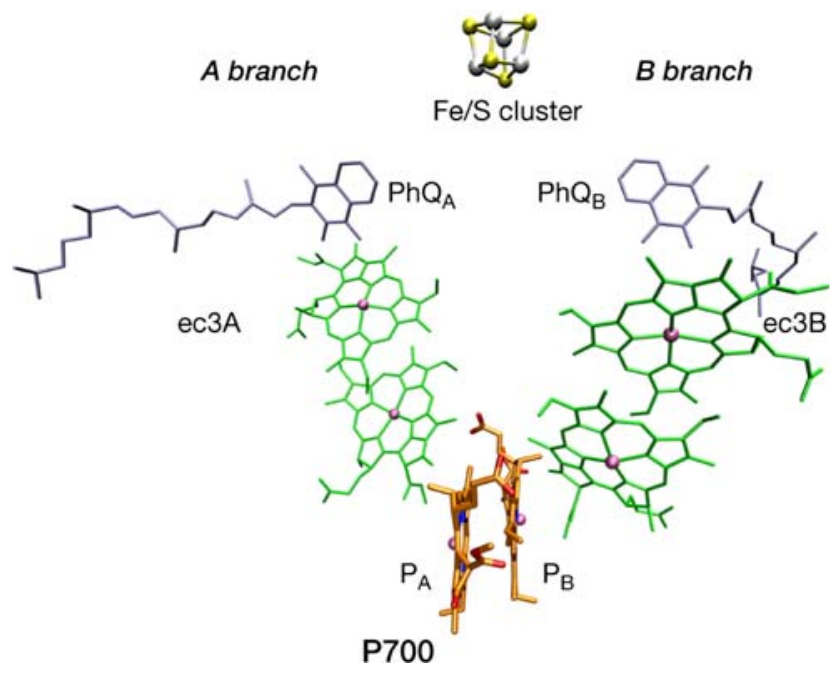

Fig. 2 A view of the electron-transfer chain in the reaction center of photosystem I. Chlorophyll pairs are arranged in two symmetric branches that diverge at P700 and reconverge at the iron-sulfur cluster. TD-DFT calculations have probed the nature of the excitation at the P700 pair that comprise P700. The detailed analysis of the individual excitations in terms of molecular orbital contributions and transition dipole moments revealed that, despite the apparent symmetric disposition of its two branches of cofactors, the P700 pair is intrinsically excited in an asymmetric fashion. On the basis of the TD-DFT results the authors were further able to establish connections with the experimentally observed asymmetric electron transfer process in PSI and propose a charge separation mechanism for P700 (Sun et al. 2007).

\section{X-ray absorption spectroscopy}

$\mathrm{X}$-ray absorption spectroscopy (XAS) is a powerful probe of the electronic and geometric structure of metal sites in inorganic and biological systems since it provides valuable information on the oxidation state, geometry, and, in some cases, spin state of the metal centre (Roe et al. 1984; Westre et al. 1997). The shape, position, and intensity of absorption peaks in the X-ray absorption near-edge structure (XANES) of the metal result from core electron excitations to valence orbitals below the ionization threshold and carry information on the oxidation state, coordination, and character of the bonding with the ligands. As with optical spectra, TDDFT can be used for the computation of metal or ligand preedge features, by allowing excitations into the virtual orbital space only out of localized core-holes (Ray et al. 2007; DeBeer George et al. 2008a). Although absolute transition energies are not predicted accurately, this simple and effective protocol yields relative transition energies for a series of related complexes or for a sequence of transitions to within a few tenths of an electron volt (DeBeer George et al. 2008a; Neese 2008a).

Applications to inorganic molecules have shown that good agreement between theory and experiment can be achieved with TD-DFT calculations using the BP86 functional in conjunction with a large decontracted doubly polarized triple- $\zeta$ basis set for the metal (Neese 2002) and polarized triple- $\zeta$ basis set for the remaining atoms (Ray et al. 2007; DeBeer George et al. 2008a, b). With relevance to potential catalytic intermediates involved in the water oxidation chemistry of PSII, Yano et al. (2007) have successfully correlated TD-DFT and experimental pre-edge spectra (1s to $3 \mathrm{~d}$ excitations) of mononuclear $\mathrm{Mn}(\mathrm{V})$ nitrido and oxo compounds. More recently, Jaszewski et al. (2008) performed TD-DFT calculations of Mn core excitations in a series of $\mathrm{Mn}$ complexes with nitrogen and oxygen donor ligands. Excitations were allowed not only from $1 \mathrm{~s}$ but also from $2 p$ orbitals, yielding results that could be compared with $1 \mathrm{~s} 2 \mathrm{p}$ resonant inelastic X-ray scattering (RIXS) studies. The computed values at the BP86/TZP level were found to agree well with the experimental correlation between $\mathrm{Mn}$ oxidation state and the $\mathrm{Mn}$ 
$\mathrm{K}$-edge and L-edge energies, confirming that TD-DFT is a robust method for analysis of XAS features. It remains to be seen how this approach extends to larger clusters such as the OEC.

\section{Mössbauer spectroscopy}

Mössbauer spectroscopy is an invaluable spectroscopic technique in bioinorganic chemistry, since it is able to probe selectively the charge and spin distribution around iron centers (Gütlich et al. 1978; see also, the contribution by Krebs and Bollinger in the present issue). The combination of DFT calculations with ${ }^{57} \mathrm{Fe}$-Mössbauer spectroscopy has emerged as a particularly fruitful strategy for the study of the ground-state properties of iron-containing enzymes (Schünemann and Winkler 2000; Gütlich and Ensling 1999). In the zero-applied magnetic field, the two main quantities that are extracted for a given iron site are the quadrupole splitting $\left(\Delta E_{\mathrm{Q}}\right)$ and the isomer shift $(\delta)$. Both quantities are related to the total electron density and are sensitive reporters of the spin state, valence state, and covalency of iron sites. The estimation of $\Delta E_{\mathrm{Q}}$ requires the calculation of the electric gradient field at the iron nucleus, which can be done with basis sets of sufficient flexibility in the core region (Neese 2002). Many studies at the B3LYP level have demonstrated that the sign and the magnitude of $\Delta E_{\mathrm{Q}}$ is predicted accurately, although absolute errors ranging from 0.3 to $1.00 \mathrm{~mm} \mathrm{~s}^{-1}$ are not uncommon (Berry et al. 2008; Godbout et al. 1999; Han et al. 2006; Salzmann et al. 1999; Sinnecker et al. 2005). Moreover, it has been shown that the computed $\Delta E_{\mathrm{Q}}$ values react fairly sensitively to details of the surrounding, such as counter ions.

The isomer shift is known from basic principles to be directly proportional to the electron density at the iron nucleus. Thus, it can be determined to good accuracy (often better than $0.1 \mathrm{~mm} \mathrm{~s}^{-1}$ ) from ground-state DFT calculations using a suitable method-specific calibration procedure on the basis of a linear correlation between the calculated electron density at the nucleus versus the measured $\delta$ (Han et al. 2006; Liu et al. 2003; Neese 2002; Sinnecker et al. 2005; Zhang et al. 2002). The predicted $\delta$ is in good agreement with experimental data when using B3LYPcalibrated curves in combination with basis sets that are flexible in the core region and extensively polarized (Berry et al. 2008; Schoneboom et al. 2005; Sinnecker et al. 2005).

\section{Exchange couplings}

In the case of bioinorganic systems which contain two or more interacting open-shell magnetic ions, the interaction is typically described in terms of the phenomenological Heisenberg-Dirac-van Vleck Hamiltonian. Thus, the main problem from the theoretical point of view becomes the evaluation of the exchange coupling constants $(J)$ that measure the "strength" of the supposed interactions between local spins. Such systems are presently handled in the DFT framework by the broken symmetry (BS) approach, which gives access to exchange coupling constants, geometries, and total energies (Noodleman 1981). Experience indicates that hybrid functionals such as B3LYP may be slightly more accurate than GGAs for the prediction of exchange coupling constants. The finer details on the procedure are a subject of ongoing controversy, but among the different formalisms to extract the $J$ values from separate high-spin and BS calculations, Yamaguchi's method appears to be most suitable since it correctly reproduces the limit of both weak and strong interaction (Yamaguchi et al. 1986). It is worth emphasizing that the BS method provides excellent electron densities owing to the variational adjustment of the ionic and neutral components of the wavefunction (Neese 2004). Therefore, this approach should be able to predict geometries that faithfully reflect those of the true low-spin states. On the other hand, the spin density remains unphysical and thus for the prediction of magnetic properties based on the BS-DFT approach, it is mandatory to use spin-projection techniques (Mouesca et al. 1995; Sinnecker et al. 2004). Several computational studies of biomimetic oxomanganese complexes have been dedicated to the prediction of $J$ values and valuable correlations between theory and experiment were found on the basis of BS-DFT calculations (Sinnecker et al. 2004, 2006).

On extension to oligonuclear systems, complications in the application of BS-DFT might arise due to the inherent indeterminacy in the values of the exchange coupling parameters. In a recent contribution (Pantazis et al. 2009), we investigate the magnetic properties of a tetramanganese complex bearing resemblance to the OEC of PSII (Fig. 3). Our results reveal that the absolute values of the exchange coupling constants $J$ are not a safe criterion for comparing theory and experiment owing to their indeterminacy when more than a few interactions among the metals exist. Instead, one should use the $J$ values computed with BSDFT to extract the actual energies of the magnetic levels by diagonalizing the Hamiltonian. These energy levels can subsequently be used for constructing magnetic susceptibility curves and form a more physically meaningful basis for comparison with experimental data. Following this approach, the energetic levels computed with the TPSSh hybrid meta-GGA functional are found to agree well with experiment despite discrepancies in the fitted exchange coupling constants. Similar observations were made by Cauchy et al. (2008) in their study of a pentanuclear iron complex. The authors point out that many different sets of $J$ values can reproduce the experimental data and proceed to exact diagonalization of the Hamiltonian and construction of a theoretical magnetic susceptibility curve to make 


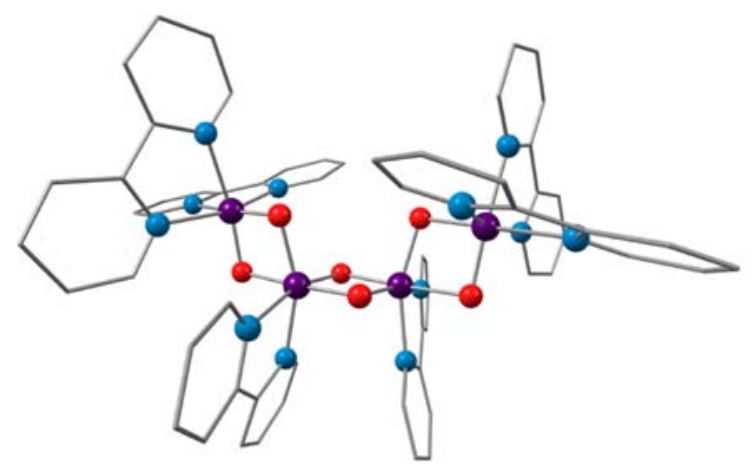

Fig. 3 The tetranuclear manganese complex $\left[\mathrm{Mn}_{4} \mathrm{O}_{6}(\text { bipyridine })_{6}\right]^{4+}$ and magnetic susceptibility curves constructed from BS-DFT results with various functionals. A direct comparison of computed and

comparisons to experiment. This approach clearly emerges as the only credible way of studying magnetic interactions with BS-DFT in oligonuclear clusters similar to the oxygen evolving complex in PSII (Pantazis et al. 2009).

\section{EPR spectroscopy}

Electron paramagnetic resonance (EPR) spectra are parameterized in terms of an effective spin Hamiltonian (SH) which contains adjustable numerical parameters that are fitted to experiments. These SH parameters are the $g$-tensor, the zero-field splitting (ZFS), and the hyperfine coupling (HFC). The accuracy of EPR parameter calculations with DFT is somewhat variable. For organic radicals and biradicals (including amino acid radicals) usually good results are obtained for the $g$-tensor, the hyperfine and quadrupole coupling and also for the ZFS (Neese 2008b). In all DFT investigations of EPR parameters specifically developed basis sets with extra flexibility in the core region such as Barone's EPR-II and EPR-III (Barone 1997) or the CP(PPP) basis sets (Neese 2002) should be employed. As regards the choice of functional, it is by now established that hybrid functionals are more accurate than GGA functionals (Neese 2008a). For transition metal complexes, the situation turns out to be more complicated. The $g$ values are usually underestimated by standard functionals, and errors of a factor of two are not uncommon. The performance of different density functionals is similar although hybrid functionals like B3LYP tend again to be slightly more accurate than GGAs like BP86 (Neese 2001a). The modeling of ZFS parameters with DFT is particularly difficult owing to the complicated spin dependence of this property (Neese 2006b). For transition metal complexes, it was shown that DFT predicts the ZFS parameter with the correct sign but tends to underestimate its magnitude, often by a factor of 2 (Neese 2003). Meanwhile, a certain number of applications have demonstrated the usefulness of ab initio treatments for the

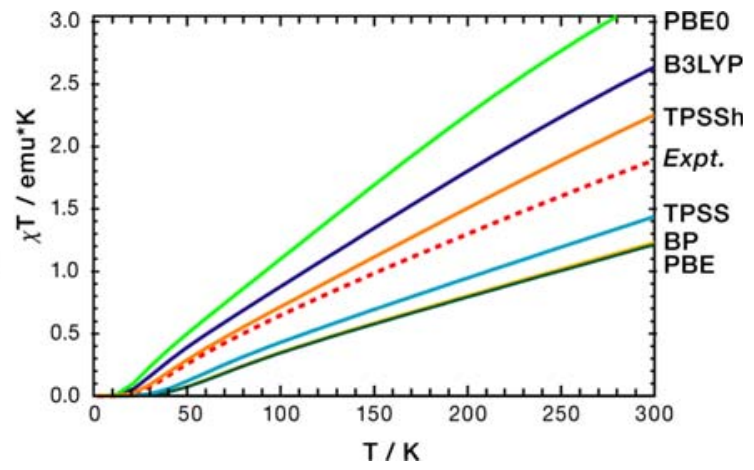

experimentally fitted exchange coupling constants is not meaningful for such systems owing to the indeterminacy of the exchange parameters

calculation of the ZFS (Ganyushin and Neese 2006; Neese et al. 2007b).

The intricacies in the application of DFT in this area are highlighted in a detailed evaluation of DFT performance for the prediction of ZFS in Mn(II) coordination complexes (Zein et al. 2008b). The study revealed that regardless of whether the spin-orbit coupling (SOC) part of the ZFS was estimated with the Pederson-Khanna or the quasi-restricted orbitals approach, accounting for the spin-spin (SS) interaction always improves the results. The physical necessity of accounting for the SS interaction is shown from its $30 \%$ contribution to the axial $D$ parameters. In general, the calculations were found to overestimate systematically the experimental $D$ values by $60 \%$. The authors call attention to the fact that the signs of calculated axial ZFS parameters are unreliable once $E / D>0.2$. Calculated $D$ and $E / D$ values were found to be highly sensitive to small structural changes; disconcertingly, the use of optimized geometries was found to lead to a significant deterioration of theoretical predictions relative to experimental XRD geometries. A subsequent study (Zein and Neese 2008) showed that using the coupled-perturbed spin-orbit coupling (CP-SOC) approach (Neese 2007) together with hybrid DFT functionals leads to a slope of the correlation line between experimental and calculated $D$ values that is essentially unity, provided that the direct SS interaction is properly included in the treatment.

For the case of the hyperfine coupling to the metal, DFT performance is not entirely satisfactory (Munzarova and Kaupp 1999; Munzarova et al. 2000). Since this property involves three contributions (Fermi contact, spin-dipolar, and spin-orbit coupling) which feature different physical mechanisms, it is difficult to calculate all of them simultaneously with quantitative accuracy. Ligand HFCs are easier to compute but, again, results are less accurate than for organic radicals, and errors of $30 \%$ must be tolerated (Neese 2001b). Kossmann et al. (2007) investigated the performance of modern DFT functionals for the prediction 
of molecular hyperfine couplings in extended test calculations for a series of small radicals and transition metal complexes. It was shown that for the prediction of metal and ligand HFCs, TPSS is better than BP86, but more importantly, that the hybrid variant TPSSh is significantly superior to TPSS and probably even better than the "de facto standard" B3LYP functional. The double-hybrid B2PLYP functional also affords accurate predictions, particularly for HFCs of metal nuclei, but the existence of outliers suggests that this method may lack stability. The reliable performance of the TPSSh functional has since received additional confirmation in our recent study (Pantazis et al. 2009) aimed at the analysis of hyperfine coupling parameters in tetramanganese models of the OEC.

A topical application of DFT for the determination of EPR parameters, highlighting the capabilities of the broken-symmetry approach, was the study of the trappedvalence, exchange-coupled $\left[\mathrm{Mn}^{\mathrm{III}} \mathrm{Mn}^{\mathrm{IV}}(\mu-\mathrm{O}) 2(\mu\right.$-OAc $)$ DTNE $]^{2+}$ complex (Sinnecker et al. 2006). Interestingly, BP86-optimized geometries were better than those obtained from B3LYP; however, B3LYP yielded exchange coupling constants in excellent agreement with experiment. The coupled perturbed Kohn-Sham equations were employed for the $g$-tensor calculations, and a strategy for the computation of $g$-tensor site values was presented that provided single-site $g$-tensors in good agreement with the expectations for the respective Mn formal oxidation states. Spin projection gave the $g$-tensor of the coupled manganese complex in good agreement with the experimental results. Small values were found for the nuclear quadrupole splitting of ${ }^{55} \mathrm{Mn}$. Hyperfine tensors were furthermore calculated and spin-projected. ${ }^{14} \mathrm{~N}$ and ${ }^{1} \mathrm{H}$ ligand hyperfine data were found to compare well with experiment. ${ }^{55} \mathrm{Mn}$ HFCs were qualitatively in line with experimental results, tracing the source of anisotropy to the $\mathrm{Mn}^{\mathrm{III}}$ center. However, isotropic ${ }^{55} \mathrm{Mn}$ HFCs were distinctly underestimated. The authors indicated that this deficiency is systematic in character and does not originate from the broken symmetry approach. Similar deviations were found between theory and experiment for DFT calculations on mononuclear Mn complexes, suggesting that the use of a universal scaling factor of approximately 1.5 might be appropriate.

\section{Summary and perspectives}

Density functional theory methods have already been established as a valuable research tool both in independent applications and as a complement of experimental investigations. In favorable cases, the calculated properties are sufficiently accurate to discriminate between structural alternatives for reaction intermediates or other species that are not amenable to experimental structure elucidation.
DFT appears generally reliable for geometries, vibrational frequencies, and total energies, having over wavefunctionbased methods the advantage of quick convergence to the basis set limit. DFT appears to be quite successful for the prediction of molecular properties as well, since a number of spectroscopic properties of interest to the bioinorganic community can be predicted with good accuracy. Hybrid functionals are in most cases better performers, with the TPSSh functional emerging as a potential new standard. There are still cases, however, where quantitative accuracy may be difficult to achieve, especially for the prediction of EPR parameters or optical spectra, necessitating a cautious and critical approach from the part of the researcher.

It is important for both practitioners of DFT and the nontechnical audience of DFT studies to keep in mind that errors do arise and they can be significant. Despite the enormous advances in density functional implementations and the sufficiently documented accuracy of results for many applications, there is no systematic way of improving DFT or converging its results to the "correct" answer, in contrast to some of the traditional wavefunction-based methods. Moreover, the success of a particular functional in one setting does not guarantee its performance in a different one. Therefore, to enhance their credibility, DFT applications must include some form of validation or estimation of the error range on the basis of careful comparison between calculated and measured observables.

A final point of interest is that DFT studies of bioinorganic systems have usually employed simplified models in vacuo. Therefore, the issue of modeling the interaction of the active site with the protein environment and the solvent comes into play (Noodleman and Han 2006; Noodleman et al. 2004, Schoneboom et al. 2005). A realistic and computationally feasible modeling of these effects can be achieved at present by combining the DFT treatment of the active site with a classical force-field description of the surrounding protein. This is the concept behind quantum mechanics/molecular mechanics (QM/MM) approaches (Senn and Thiel 2007), which are discussed by Batista and coworkers in the present issue. In a broader theoretical context, many issues can be identified that warrant further developments. We anticipate that in the future we will witness developments regarding functionals that provide a consistent treatment of exact exchange, improvements in the treatment of electronic relaxation and excited states, and a more proper treatment of magnetic and relativistic effects. A longer term target is certainly the reliable, consistent and efficient treatment of system dynamics or of very large systems.

Acknowledgements We gratefully acknowledge financial support of our research from the German Science Foundation (SPP 1137) and the Max-Planck Society via a Max-Planck Fellowship arrangement 
for FN. We are indebted to Prof. Wolfgang Lubitz and Prof. Johannes Messinger for stimulating discussions about photosystem II and the EPR spectroscopic properties of oligonuclear manganese clusters. We also thank Dr. Taras Petrenko for his theoretical contributions to metal cluster magnetic properties and Dr. Frank Wennmohs, Ms. Ute Becker, Mr. Rolf Trinoga and Mr. Jens Mekelburger, for valuable technical assistance.

Open Access This article is distributed under the terms of the Creative Commons Attribution Noncommercial License which permits any noncommercial use, distribution, and reproduction in any medium, provided the original author(s) and source are credited.

\section{References}

Baerends EJ, Ellis DE, Ros P (1973) Self-consistent molecular Hartree-Fock-Slater calculations-I. The computational procedure. Chem Phys 2:41-51. doi:10.1016/0301-0104(73)80059-X

Barone V (1997) Recent advances in density functional methods, part I. In: Chong DP (ed) World Scientific, Singapore

Bauernschmitt R, Ahlrichs R (1996) Treatment of electronic excitations within the adiabatic approximation of time dependent density functional theory. Chem Phys Lett 256:454-464. doi: 10.1016/0009-2614(96)00440-X

Becke AD (1988) Density-functional exchange-energy approximation with correct asymptotic behavior. Phys Rev A 38:3098-3100. doi:10.1103/PhysRevA.38.3098

Becke AD (1993) A new mixing of Hartree-Fock and local densityfunctional theories. J Chem Phys 98:1372-1377. doi:10.1063/ 1.464304

Berry JF, DeBeer George S, Neese F (2008) Electronic structure and spectroscopy of "superoxidized" iron centers in model systems: theoretical and experimental trends. Phys Chem Chem Phys 10:4361-4374. doi:10.1039/b801803k

Bühl M, Reimann C, Pantazis DA, Bredow T, Neese F (2008) Geometries of third-row transition-metal complexes from density functional theory. J Chem Theory Comput 4:1449-1459. doi: $10.1021 /$ ct800172j

Casida ME, Jamorski C, Casida KC, Salahub DR (1998) Molecular excitation energies to high-lying bound states from timedependent density-functional response theory: characterization and correction of the time-dependent local density approximation ionization threshold. J Chem Phys 108:4439-4449. doi: $10.1063 / 1.475855$

Cauchy T, Ruiz E, Alvarez S (2008) Exchange interactions in a $\mathrm{Fe}_{5}$ complex: a theoretical study using density functional theory. Inorg Chim Acta 361:3832-3835. doi:10.1016/j.ica. 2008.02.011

DeBeer George S, Petrenko T, Neese F (2008a) A simple timedependent density functional theory based protocol for the prediction of X-ray absorption spectra. I. Ligand K-edges. Inorg Chim Acta 361:965-972. doi:10.1016/j.ica.2007.05.046

DeBeer George S, Petrenko T, Neese F (2008b) Prediction of iron Kedge absorption spectra using time-dependent density functional theory. J Phys Chem A doi:10.1021/jp803174m

Eichkorn K, Weigend F, Treutler O, Ahlrichs R (1997) Auxiliary basis sets for main row atoms and transition metals and their use to approximate Coulomb potentials. Theor Chem Acc 97:119124. doi: $10.1007 / \mathrm{s} 002140050244$

Fiedler AT, Bryngelson PA, Maroney MJ, Brunold TC (2005) Spectroscopic and computational studies of $\mathrm{Ni}$ superoxide dismutase: electronic structure contributions to enzymatic function. J Am Chem Soc 127:5449-5462. doi:10.1021/ja042521i
Ganyushin D, Neese F (2006) First-principles calculations of zerofield splitting parameters. J Chem Phys 125:024103. doi: 10.1063/1.2213976

Ganyushin D, Neese F (2008) First-principles calculations of magnetic circular dichroism spectra. J Chem Phys 128:114117. doi:10.1063/1.2894297

Gascon JA, Sproviero EM, McEvoy JP, Brudvig GW, Batista VS (2007) Ligation of the C-terminus of the D1-polypeptide of photosystem II to the oxygen evolving complex of photosystem II. In: Allen JF, Gautt E, Golbeck JH, Osmond B (eds) Photosynthesis. Energy from the sun. 14th international congress on photosynthesis, pp 363-368, Springer, Dordrecht, The Netherlands

Godbout N, Sanders LK, Salzmann R, Havlin RH, Wojdelski M, Oldfield E (1999) Solid-state NMR, Mössbauer, crystallographic, and density functional theory investigation of $\mathrm{Fe}-\mathrm{O}_{2}$ and $\mathrm{Fe}-\mathrm{O}_{2}$ analogue metalloporphyrins and metalloproteins. J Am Chem Soc 121:3829-3844. doi:10.1021/ja9832820

Grapperhaus CA, Bill E, Weyhermuller T, Neese F, Wieghardt K (2001) Molecular and electronic structure of $\left[\mathrm{Mn}^{\mathrm{V}} \mathrm{N}(\right.$ cyclamacetato) $\mathrm{PF}_{6}$. A combined experimental and DFT study. Inorg Chem 40:4191-4198. doi:10.1021/ic001370r

Grimme S (2006a) Semiempirical hybrid density functional with perturbative second-order correlation. J Chem Phys 124:34108. doi: $10.1063 / 1.2148954$

Grimme S (2006b) Semiempirical GGA-type density functional constructed with a long-range dispersion correction. J Comput Chem 27:1787-1799. doi:10.1002/jcc.20495

Gritsenko OV, Schipper PRT, Baerends EJ (1999) Approximation of the exchange-correlation Kohn-Sham potential with a statistical average of different orbital model potentials. Chem Phys Lett 302:199-207. doi:10.1016/S0009-2614(99)00128-1

Gütlich P, Ensling J (1999) Inorganic electronic structure and spectroscopy. Wiley, New York

Gütlich P, Link R, Trautwein A (1978) Mössbauer spectroscopy and transition metal chemistry. Springer, Heidelberg

Han W-G, Liu T, Lovell T, Noodleman L (2006) DFT calculations of ${ }^{57} \mathrm{Fe}$ Mössbauer isomer shifts and quadrupole splittings for iron complexes in polar dielectric media: applications to methane monooxygenase and ribonucleotide reductase. J Comput Chem 27:1292-1306. doi:10.1002/jcc.20402

Hohenberg P, Kohn W (1964) Inhomogeneous electron gas. Phys Rev B 136:864-1138. doi:10.1103/PhysRev.136.B864

Jackson TA, Karapetian A, Miller AF, Brunold TC (2005) Probing the geometric and electronic structures of the low-temperature azide adduct and the product-inhibited form of oxidized manganese superoxide dismutase. Biochemistry 44:1504-1520. doi:10.1021/bi048639t

Jaszewski AR, Stranger R, Pace RJ (2008) Time-dependent DFT studies of metal core-electron excitations in Mn complexes. J Phys Chem A 112:11223-11234. doi:10.1021/jp803286c

Jensen KP (2008) Bioinorganic chemistry modeled with the TPSSh density functional. Inorg Chem Inorg Chem 47:10357-10365. doi:10.1021/ic800841t

Koch W, Holthausen MC (2000) A chemist's guide to density functional theory. Wiley-VCH, Weinheim

Kohn W, Sham LJ (1965a) Quantum density oscillations in an inhomogeneous electron gas. Phys Rev A 137:1697-1705. doi: 10.1103/PhysRev.137.A1697

Kohn W, Sham LJ (1965b) Self-consistent equations including exchange and correlation effects. Phys Rev A 140:1133-1138. doi:10.1103/PhysRev.140.A1133

Kossmann S, Kirchner B, Neese F (2007) Performance of modern density functional theory for the prediction of hyperfine structure: meta-GGA and double hybrid functionals. Mol Phys 105:2049-2071. doi:10.1080/00268970701604655 
Lee C, Yang W, Parr RG (1988) Development of the Colle-Salvetti correlation-energy formula into a functional of the electron density. Phys Rev B 37:785-789. doi:10.1103/PhysRevB.37.785

Liu T, Lovell T, Han WG, Noodleman L (2003) DFT calculations of isomer shifts and quadrupole splitting parameters in synthetic iron-oxo complexes: applications to methane monooxygenase and ribonucleotide reductase. Inorg Chem 42:5244-5251. doi: 10.1021/ic020640y

Lundberg M, Siegbahn PEM (2004) Theoretical investigations of structure and mechanism of the oxygen-evolving complex in PSII. Phys Chem Chem Phys 6:4772-4780. doi:10.1039/ b406552b

Mouesca JM, Noodleman L, Case DA, Lamotte B (1995) Spin densities and spin coupling in iron-sulfur clusters: a new analysis of hyperfine coupling constants. Inorg Chem 34:4347-4359. doi: 10.1021/ic00121a013

Munzarova M, Kaupp M (1999) A critical validation of density functional and coupled-cluster approaches for the calculation of EPR hyperfine coupling constants in transition metal complexes. J Phys Chem A 103:9966-9983. doi:10.1021/jp992303p

Munzarova ML, Kubacek P, Kaupp M (2000) Mechanisms of EPR hyperfine coupling in transition metal complexes. J Am Chem Soc 122:11900-11913. doi:10.1021/ja002062v

Murray CW, Laming GJ, Handy NC, Amos RD (1992) Kohn-Sham bond lengths and frequencies calculated with accurate quadrature and large basis-sets. Chem Phys Lett 199:551-556. doi: 10.1016/0009-2614(92)85008-X

Neese F (2001a) Prediction of electron paramagnetic resonance $g$ values using coupled perturbed Hartree-Fock and Kohn-Sham theory. J Chem Phys 115:11080-11096. doi:10.1063/1.1419058

Neese F (2001b) Theoretical study of ligand superhyperfine structure application to $\mathrm{Cu}(\mathrm{II})$ complexes. J Phys Chem A 105:42904299. doi:10.1021/jp003254f

Neese F (2002) Prediction and interpretation of the ${ }^{57} \mathrm{Fe}$ isomer shift in Mössbauer spectra by density functional theory. Inorg Chim Acta 337:181-192. doi:10.1016/S0020-1693(02)01031-9

Neese F (2003) Quantum chemical calculations of spectroscopic properties of metalloproteins and model compounds: EPR and Mössbauer properties. Curr Opin Chem Biol 7:125-135. doi: 10.1016/S1367-5931(02)00006-6

Neese F (2004) Definition of corresponding orbitals and the diradical character in broken symmetry DFT calculations on spin coupled systems. J Phys Chem Solids 65:781-785. doi:10.1016/j.jpcs. 2003.11.015

Neese F (2006a) A critical evaluation of DFT including timedependent DFT, applied to bioinorganic chemistry. J Biol Inorg Chem 11:702-711. doi:10.1007/s00775-006-0138-1

Neese F (2006b) Importance of direct spin-spin coupling and spin-flip excitations for the zero-field splittings of transition metal complexes: a case study. J Am Chem Soc 128:10213-10222. doi:10.1021/ja061798a

Neese F (2007) Calculation of the zero-field splitting tensor on the basis of hybrid density functional and Hartree-Fock theory. J Chem Phys 127:164112. doi:10.1063/1.2772857

Neese F (2008a) Calculation of molecular spectra and molecular properties with density functional theory: from fundamental theory to exchange coupling. Coord Chem Rev doi:10.1016/ j.ccr.2008.05.014

Neese F (2008b) Spin Hamiltonian parameters from first principle calculations: theory and application. In: Hanson G, Berliner L (eds) High resolution EPR: applications to metalloenzymes and metals in medicine. Biological magnetic resonance, vol 28. Springer, Berlin, pp 175-232

Neese F, Schwabe T, Grimme S (2007a) Analytic derivatives for perturbatively corrected "double hybrid" density functionals: theory, implementation, and applications. J Chem Phys 126:124115. doi: $10.1063 / 1.2712433$

Neese F, Petrenko T, Ganyushin D, Olbrich G (2007b) Advanced aspects of $a b$ initio theoretical optical spectroscopy of transition metal complexes: multiplets, spin-orbit coupling and resonance Raman intensities. Coord Chem Rev 251:288-327. doi:10.1016/ j.ccr.2006.05.019

Neese F, Wennmohs F, Hansen A, Becker U (2008) Efficient, approximate and parallel Hartree-Fock and hybrid DFT calculations. A 'Chain-of-Spheres' algorithm for the Hartree-Fock exchange. Chem Phys doi:10.1016/j.chemphys.2008.10.036

Neugebauer J, Hess BA (2003) Fundamental vibrational frequencies of small polyatomic molecules from density-functional calculations and vibrational perturbation theory. J Chem Phys 118:7215-7225. doi:10.1063/1.1561045

Noodleman L (1981) Valence bond description of antiferromagnetic coupling in transition metal dimers. J Chem Phys 74:5737-5743. doi:10.1063/1.440939

Noodleman L, Han WG (2006) Structure, redox, pK(a), spin. A golden tetrad for understanding metalloenzyme energetics and reaction pathways. J Biol Inorg Chem 11:674-694. doi: 10.1007/s00775-006-0136-3

Noodleman L, Lovell T, Han WG, Li J, Himo F (2004) Quantum chemical studies of intermediates and reaction pathways in selected enzymes and catalytic synthetic systems. Chem Rev 104:459-508. doi:10.1021/cr020625a

Pantazis DA, Orio M, Petrenko T, Zein S, Bill E, Lubitz W, Messinger J, Neese F (2009) A new quantum chemical approach to the magnetic properties of oligonuclear transition metal clusters: application to a model for the tetranuclear manganese cluster of photosystem II. Chem Eur J. doi:10.1002/chem. 200802456

Parr RG, Yang W (1989) Density functional theory of atoms and molecules. Oxford University Press, Oxford

Perdew JP, Burke K, Ernzerhof M (1996) Generalized gradient approximation made simple. Phys Rev Lett 77:3865-3868. doi: 10.1103/PhysRevLett.77.3865

Ray K, DeBeer George S, Solomon E, Wieghardt K, Neese F (2007) Description of the ground-state covalencies of the bis(dithiolato) transition-metal complexes from X-ray absorption spectroscopy and time-dependent density-functional calculations. Chem Eur J 13:2783-2797. doi:10.1002/chem.200601425

Roe AL, Schneider DJ, Mayer RJ, Pyrz JW, Widom J, Que L (1984) $\mathrm{X}$-ray absorption spectroscopy of iron-tyrosinate proteins. J Am Chem Soc 106:1676-1681. doi:10.1021/ja00318a021

Salzmann R, McMahon MT, Godbout N, Sanders LK, Wojdelski M, Oldfield E (1999) Solid-state NMR, crystallographic and density functional theory investigation of $\mathrm{Fe}-\mathrm{CO}$ and $\mathrm{Fe}-\mathrm{CO}$ analogue metalloporphyrins and metalloproteins. J Am Chem Soc 121:3818-3828. doi:10.1021/ja9832818

Schenker R, Mock MT, Kieber-Emmons MT, Riordan CG, Brunold TC (2005) Spectroscopic and computational studies on [Ni(tmc)$\mathrm{CH}_{3}$ ]OTf: implications for Ni-methyl bonding in the A cluster of acetyl-CoA synthase. Inorg Chem 44:3605-3617. doi:10.1021/ ic0483996

Schoneboom JC, Neese F, Thiel W (2005) Toward identification of the compound I reactive intermediate in cytochrome P450 chemistry: A QM/MM study of its EPR and Mössbauer parameters. J Am Chem Soc 127:5840-5853. doi:10.1021/ ja0424732

Schünemann V, Winkler H (2000) Structures and dynamics of biomolecules studied by Mössbauer spectroscopy. Rep Prog Phys 63:263-353. doi:10.1088/0034-4885/63/3/202

Senn HM, Thiel W (2007) QM/MM methods for biological systems. Top Curr Chem 268:173-290. doi:10.1007/128_2006_084 
Seth M, Ziegler T (2006) Calculation of excitation energies of openshell molecules with spatially degenerate ground states. II. Transformed reference via intermediate configuration KohnSham time dependent density functional theory oscillator strengths and magnetic circular dichroism C terms. J Chem Phys 124:144105. doi:10.1063/1.2187004

Seth M, Ziegler T, Banerjee A, Autschbach J, van Gisbergen SJA, Baerends EJ (2004) Calculation of the A term of magnetic circular dichroism based on time dependent-density functional theory I. Formulation and implementation. J Chem Phys 120:10942-10954. doi:10.1063/1.1747828

Seth M, Ziegler T, Autschbach J (2005) Ab initio calculation of the C/ $\mathrm{D}$ ratio of magnetic circular dichroism. J Chem Phys 122:094112. doi:10.1063/1.1856453

Siegbahn PEM (2003) Mechanisms of metalloenzymes studied by quantum chemical methods. Q Rev Biophys 36:91-145. doi: $10.1017 / \mathrm{S} 0033583502003827$

Siegbahn PEM (2006a) O-O bond formation in the $\mathrm{S}_{4}$ state of the oxygen-evolving complex in photosystem II. Chem Eur J 12:9217-9227. doi:10.1002/chem.200600774

Siegbahn PEM (2006b) The performance of hybrid DFT for mechanisms involving metal complexes in enzymes. J Biol Inorg Chem 11:695-701. doi:10.1007/s00775-006-0137-2

Siegbahn PEM (2008a) Theoretical studies of O-O bond formation in photosystem II. Inorg Chem 47:1779-1786. doi:10.1021/ ic7012057

Siegbahn PEM (2008b) A structure-consistent mechanism for dioxygen formation in photosystem II. Chem Eur J 14:8290-8302. doi:10.1002/chem. 200800445

Sinnecker S, Neese F, Noodleman L, Lubitz W (2004) Calculating the electron paramagnetic resonance parameters of exchange coupled transition metal complexes using broken symmetry density functional theory: application to a $\mathrm{Mn}^{\mathrm{III}} / \mathrm{Mn}^{\mathrm{IV}}$ model compound. J Am Chem Soc 126:2613-2622. doi:10.1021/ ja0390202

Sinnecker S, Slep LD, Bill E, Neese F (2005) Performance of nonrelativistic and quasi-relativistic hybrid DFT for the prediction of electric and magnetic hyperfine parameters in ${ }^{57} \mathrm{Fe}$ Mössbauer spectra. Inorg Chem 44:2245-2254. doi:10.1021/ ic048609e

Sinnecker S, Neese F, Lubitz W (2006) Dimanganese catalasespectroscopic parameters from broken-symmetry density functional theory of the superoxidized $\mathrm{Mn}^{\mathrm{III}} / \mathrm{Mn}^{\mathrm{IV}}$ state. J Biol Inorg Chem 10:231-238. doi:10.1007/s00775-005-0633-9

Sosa C, Andzelm J, Elkin BC, Wimmer E, Dobbs KD, Dixon DA (1992) A local density functional-study of the structure and vibrational frequencies of molecular transition-metal compounds. J Phys Chem 96:6630-6636. doi:10.1021/j100195a022

Sproviero EM, Shinopoulos K, Gascon JA, McEvoy JP, Brudvig GW, Batista VS (2007) QM/MM computational studies of substrate water binding to the oxygen-evolving centre of photosystem II. Philos Trans R Soc B 363:1149-1156. doi:10.1098/rstb. 2007.2210

Sproviero EM, Gascon JA, McEvoy JP, Brudvig GW, Batista VS (2008a) Quantum mechanics/molecular mechanics study of the catalytic cycle of water splitting in photosystem II. J Am Chem Soc 130:3428-3442. doi:10.1021/ja076130q

Sproviero EM, McEvoy JP, Gascon JA, Brudvig GW, Batista VS (2008b) Computational insights into the $\mathrm{O}_{2}$-evolving complex of photosystem II. Photosynth Res 97:91-114. doi:10.1007/s11120008-9307-0

Staroverov VN, Scuseria GE, Tao J, Perdew JP (2003) Comparative assessment of a new nonempirical density functional: molecules and hydrogen-bonded complexes. J Chem Phys 119:12-129. doi: $10.1063 / 1.1626543$

Stich TA, Buan NR, Escalante-Semerena JC, Brunold TC (2005) Spectroscopic and computational studies of the ATP:corrinoid adenosyltransferase (CobA) from Salmonella enterica: insights into the mechanism of adenosylcobalamin biosynthesis. J Am Chem Soc 127:8710-8719. doi:10.1021/ja042142p

Stratmann RE, Burant JC, Scuseria GE, Frisch MJ (1997) Improving harmonic vibrational frequencies calculations in density functional theory. J Chem Phys 106:10175-10183. doi:10.1063/ 1.474047

Stratmann RE, Scuseria GE, Frisch MJ (1998) An efficient implementation of time-dependent density-functional theory for the calculation of excitation energies of large molecules. J Chem Phys 109:8218-8224. doi:10.1063/1.477483

Sun Y, Dai Z, Wang W, Sun Y (2007) A TDDFT study on the excitation of P700. Chem Phys Lett 434:111-115. doi:10.1016/ j.cplett.2006.11.090

Szabo A, Ostlund NS (1989) Modern quantum chemistry. McGrawHill, New York

Vahtras O, Almlöf J, Feyereisen MW (1993) Integral approximations for LCAO-SCF calculations. Chem Phys Lett 213:514-518. doi: 10.1016/0009-2614(93)89151-7

Westre TE, Kennepohl P, DeWitt JG, Hedman B, Hodgson KO, Solomon EI (1997) A multiplet analysis of Fe K-edge 1s $\rightarrow$ 3d pre-edge features of iron complexes. J Am Chem Soc 119:62976314. doi:10.1021/ja964352a

Yamaguchi K, Takahara Y, Fueno T (1986) Applied quantum chemistry. In: Smith VH, Schaefer HF, Morokuma K (eds) Reidel, Dordrecht

Yano J, Kern J, Sauer K, Latimer MJ, Pushkar Y, Biesiadka J, Loll B, Saenger W, Messinger J, Zouni A, Yachandra VK (2005) Where water is oxidized to dioxygen: structure of the photosynthetic $\mathrm{Mn}_{4} \mathrm{Ca}$ cluster. Science 314:821-825. doi:10.1126/science. 1128186

Yano J, Robblee J, Pushkar Y, Marcus MA, Bendix J, Workman JM, Collins TJ, Solomon EI, DeBeer George S, Yachandra VK (2007) Polarized X-ray absorption spectroscopy of single-crystal $\mathrm{Mn}(\mathrm{V})$ complexes relevant to the oxygen-evolving complex of photosystem II. J Am Chem Soc 129:12989-13000. doi: $10.1021 / \mathrm{ja} 071286 \mathrm{~b}$

Zein S, Neese F (2008) Ab initio and coupled-perturbed density functional theory estimation of zero-field splittings in $\mathrm{Mn}^{\mathrm{II}}$ transition metal complexes. J Phys Chem A 112:7976-7983. doi: 10.1021/jp804062a

Zein S, Kulik LV, Yano J, Kern J, Zouni A, Yachandra VK, Lubitz W, Neese F, Messinger J (2008a) Focussing the view on nature's water splitting catalyst. Philos Trans R Soc B 363:1167-1177. doi:10.1098/rstb.2007.2212

Zein S, Duboc C, Lubitz W, Neese F (2008b) A systematic density functional study of the zero-field splitting in Mn(II) coordination compounds. Inorg Chem 47:134-142. doi:10.1021/ic701293n

Zhang Y, Mao J, Oldfield E (2002) ${ }^{57} \mathrm{Fe}$ Mössbauer isomer shifts of heme protein model systems: electronic structure calculations. J Am Chem Soc 124:7829-7839. doi:10.1021/ja011583v 